Changkun Chen

Department of Mechanical Science and

Bioengineering,

Graduate School of Engineering Science, Osaka University,

1-3 Machikaneyama, Toyonaka, Osaka 560-8531, Japan

e-mail: chenchangkun@mbox.me.es.osaka-u.ac.jp

Christophe Nicolet

Laboratory for Hydraulic Machines

(LMH-IMHEF),

École Polytechnique Fédérale de Lausanne

(EPFL),

Avenue de Cour 33bis,

$\mathrm{CH}-1007$ Lausanne, Switzerland

e-mail: christophe.nicolet@epfl.ch

Koichi Yonezawa

Department of Mechanical Science and Bioengineering,

Graduate School of Engineering Science, Osaka University,

1-3 Machikaneyama, Toyonaka, Osaka 560-8531, Japan

e-mail: yonezawa@me.es.osaka-u.ac.jp

Mohamed Farhat

e-mail: mohamed.farhat@epfl.ch

Francois Avellan

e-mail: francois.avellan@epfl.ch

Laboratory for Hydraulic Machines

(LMH-IMHEF),

École Polytechnique Fédérale de Lausanne

(EPFL),

Avenue de Cour 33bis,

CH-1007 Lausanne, Switzerland

\author{
Yoshinobu Tsujimoto \\ Department of Mechanical Science and \\ Bioengineering, \\ Graduate School of Engineering Science, \\ Osaka University, \\ 1-3 Machikaneyama, Toyonaka, \\ Osaka 560-8531, Japan \\ e-mail: tujimoto@me.es.osaka-u.ac.jp
}

\title{
One-Dimensional Analysis of Full Load Draft Tube Surge
}

One-dimensional stability analysis of a hydraulic system composed of a penstock, a runner, and a draft tube was carried out to determine the cause of the full load draft tube surge. It is assumed that the cavity volume at the runner exit is a function of the pressure at the vortex core evaluated from the instantaneous local pressure at the runner exit and an additional pressure decrease due to the centrifugal force on the swirling flow. It was found that the diffuser effect of the draft tube has a destabilizing effect over all flow rates, while the swirl effects stabilize/destabilize the system at larger/smaller flow rates than the swirl-free flow rate. Explanations of the destabilizing mechanism are given for the diffuser and swirl flow effects. [DOI: 10.1115/1.2903475]

\section{Introduction}

Securing stable operation is one of the most important issues in hydraulic power generation systems. At part load, a draft tube surge occurs when the frequency of the vortex rope whirl agrees with the resonant frequency of the hydraulic system [1-4]. It is also known that a surge can occur even at full load [5] and the cause is still not clear. Koutnik and Pulpitel [6] simulated the full load surge by representing the effect of the cavitation in the draft

Contributed by the Fluids Engineering Division of ASME for publication in the Journal of Fluids EngineERING. Manuscript received September 27, 2007; final manuscript received January 15, 2008; published online April 14, 2008. Assoc. Editor: Steven Ceccio. tube by using cavitation compliance $C=-\partial V_{c} / \partial p_{D}$ and mass flow gain factor $\chi=-\partial V_{c} / \partial Q_{D}$, where $V_{c}$ is the volume of the cavity and $p_{D}$ and $Q_{D}$ are the pressure and flow rate downstream of the cavity. It was shown that the instability occurs when the absolute value of the negative mass flow gain factor is larger than a certain value, which depends on the value of the cavitation compliance and system head losses. This model was combined with the numerical analysis software SIMSEN to analyze the full load surge observed in a real plant [7].

Although Refs. [6,7] show that a full load surge can be successfully simulated by using an appropriate value of the mass flow gain factor, the flow mechanism determining the value of the mass flow gain factor is not clear yet. The present study is intended to 


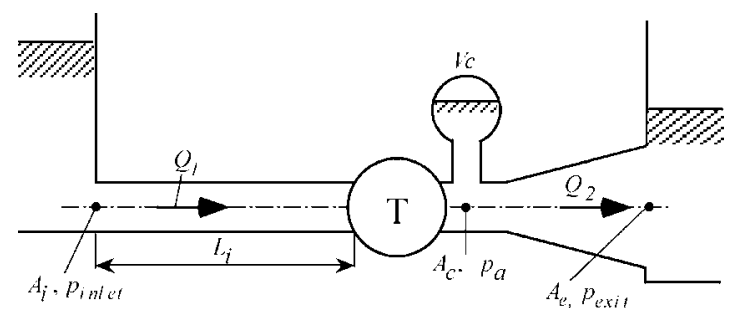

Fig. 1 Hydraulic system for the analysis

clarify the diffuser effect of the draft tube and the effects of swirl in the downstream of the runner on the hydraulic instabilities in power generation plants.

\section{Analytical Model}

We consider a system composed of an inlet pipe of length $L_{i}$ and area $A_{i}$, a turbine runner, and a draft tube with the inlet and exit areas $A_{c}$ and $A_{e}$, respectively, as shown in Fig. 1 . It is assumed that a cavity of volume $V_{c}$ appears downstream of the turbine and upstream of the draft tube. Then, from continuity, the following relation exists between upstream and downstream flow rates $Q_{1}$ and $Q_{2}$ :

$$
Q_{2}-Q_{1}=d V_{c} / d t
$$

Under constant runner speed and guide vane opening, we can represent the runner by a resistance with a constant loss coefficient $\zeta_{T}$, which depends on the guide vane opening. If we assume the incompressible flow, the following equation can be obtained from the unsteady version of Bernoulli's equation applied to the upstream pipe:

$$
p_{\text {inlet }}=p_{a}+\rho \frac{L_{i}}{A_{i}} \frac{d Q_{1}}{d t}+\rho \frac{\zeta_{T}}{2 A_{i}^{2}} Q_{1}^{2}
$$

where $p_{\text {inlet }}$ is the pressure at the pipe inlet and $p_{a}$ is the pressure at the runner discharge. The loss in the pipe can be included in $\zeta_{T}$. By applying the unsteady version of Bernoulli's equation to the draft tube, we obtain

$$
p_{a}=p_{\text {exit }}+\rho \frac{L_{e}}{A_{e}} \frac{d Q_{2}}{d t}+\rho \frac{\zeta_{2}-D}{2 A_{e}^{2}} Q_{2}^{2}
$$

where $L_{e}=\int\left(A_{e} / A(s)\right) d s$ is the effective length of the draft tube, $A_{e}$ is the exit area of the draft tube, $D=\left(A_{e} / A_{c}\right)^{2}-1$ is the diffusion factor, $A_{c}$ is the inlet area of the draft tube, and $\zeta_{2}$ is the loss coefficient of the draft tube. For simplicity, $\zeta_{2}$ is assumed to be constant although it may depend on the swirl of the discharge flow [8].

At off-design operating point, the discharge flow from the runner swirls and a vortex is formed. If the pressure $p_{c}$ at the vortex center is lower than the vapor pressure, a cavity will appear. The volume of the cavity can be considered to be a function of the core pressure $p_{c}$,

$$
V_{c}=V_{c}\left(p_{c}\right)
$$

Due to the centrifugal force on the swirling flow, the core pressure $p_{c}$ is lower than the ambient pressure $p_{a}$ and can be expressed as

$$
p_{c}=p_{a}-\rho \alpha c_{\theta 2}^{2}
$$

Here, $c_{\theta 2}$ is a representative swirl velocity and $\alpha$ is a pressure coefficient for the swirl effects. If we assume a Rankine combined vortex with the core radius $a$ and the outer radius $R, \alpha$ is determined to be $\alpha=(R / a)^{2}-1 / 2$, with $c_{\theta 2}$ evaluated at the outer radius $R$, although the real flow from the runner is much more complicated [8].

From the velocity triangle at the runner discharge, we obtain

$$
c_{\theta 2}=c_{m 2} \cot \beta_{2}-U_{2}=\frac{Q_{1}}{S} \cot \beta_{2}-U_{2}
$$

where $c_{m 2}$ is the meridional velocity at the runner exit, $\beta_{2}$ is the runner exit vane angle, $S$ is the runner exit area, and $U_{2}$ is the runner exit peripheral speed.

By putting Eqs. (3) and (6) in Eq. (5), we obtain

$$
p_{c}=p_{\text {exit }}+\rho \frac{L_{e}}{A_{e}} \frac{d Q_{2}}{d t}+\rho \frac{\zeta_{2}-D}{2 A_{e}^{2}} Q_{2}^{2}-\rho \alpha\left(\frac{\cot \beta_{2}}{S} Q_{1}-U_{2}\right)^{2}
$$

We define the cavitation compliance $C$ by

$$
C=-d V_{c} / d p_{c}
$$

Then, the continuity equation (1) can be expressed as

$$
\begin{aligned}
Q_{2}-Q_{1}= & d V_{c} / d t=\left(d V_{c} / d p_{c}\right)\left(d p_{c} / d t\right)=-C\left(d p_{c} / d t\right) \\
= & -\rho C \frac{L_{e}}{A_{e}} \frac{d^{2} Q_{2}}{d t^{2}}+\rho C \frac{D-\zeta_{2}}{A_{e}^{2}} Q_{2} \frac{d Q_{2}}{d t} \\
& +2 \rho C \alpha \frac{\cot \beta_{2}}{S}\left(\frac{\cot \beta_{2}}{S} Q_{1}-U_{2}\right) \frac{d Q_{1}}{d t}
\end{aligned}
$$

The second term with $d Q_{2} / d t$ represents the diffuser effect corresponding to the mass flow gain factor. If the discharge flow $Q_{2}$ is increased, the ambient pressure $p_{a}$ is decreased if the diffuser effect $D$ is larger than the loss $\zeta_{2}$, resulting in an increase in cavity volume. The third term with $d Q_{1} / d t$ represents the effect of swirl. This term may also be called the "mass flow gain factor" but this term is associated with the upstream flow $Q_{1}$. At flow rates higher than design $\left(Q_{1}>U_{2} S \tan \beta_{2}\right)$, the tangential velocity $c_{\theta 2}$ and the cavity volume increase as the upstream flow rate $Q_{1}$ is increased. The opposite result is obtained at a smaller flow rate.

By putting Eq. (3) in Eq. (2), we obtain

$$
p_{\text {inlet }}=p_{\text {exit }}+\rho \frac{L_{e}}{A_{e}} \frac{d Q_{2}}{d t}+\rho \frac{\zeta_{2}-D}{2 A_{e}^{2}} Q_{2}^{2}+\rho \frac{L_{i}}{A_{i}} \frac{d Q_{1}}{d t}+\rho \frac{\zeta_{T}}{2 A_{i}^{2}} Q_{1}^{2}
$$

Equations (9) and (10) are the fundamental equations in determining $Q_{1}(t)$ and $Q_{2}(t)$. For stability analysis, we assume $Q_{1}$ $=\bar{Q}_{1}+\widetilde{Q}_{1}(t), Q_{1}=\bar{Q}_{2}+\widetilde{Q}_{2}(t)$ and $\bar{Q}_{1} \gg \widetilde{Q}_{1}(t), \bar{Q}_{2} \gg \widetilde{Q}_{2}(t)$. By assuming $\bar{Q}_{1}=\bar{Q}_{2}=\bar{Q}$, the unsteady parts of Eqs. (10) and (9) can be expressed as

$$
\begin{aligned}
0=\rho \frac{L_{e}}{A_{e}} \frac{d \tilde{Q}_{2}}{d t} & +\rho \frac{\zeta_{2}-D}{A_{e}^{2}} \bar{Q} \tilde{Q}_{2}+\rho \frac{L_{i}}{A_{i}} \frac{d \tilde{Q}_{1}}{d t}+\rho \frac{\zeta_{T}}{A_{i}^{2}} \bar{Q} \tilde{Q}_{1} \\
\tilde{Q}_{2}-\widetilde{Q}_{1}= & -\rho C \frac{L_{e}}{A_{e}} \frac{d^{2} \tilde{Q}_{2}}{d t^{2}}+\rho C \frac{D-\zeta_{2}}{A_{e}^{2}} \bar{Q} \frac{d \tilde{Q}_{2}}{d t} \\
& +2 \rho C \alpha \frac{\cot \beta_{2}}{S}\left(\frac{\cot \beta_{2}}{S} \bar{Q}-U_{2}\right) \frac{d \tilde{Q}_{1}}{d t}
\end{aligned}
$$

Here, we assume $\widetilde{Q}_{1}=\widetilde{Q}_{1,0} e^{j \omega t}, \widetilde{Q}_{2}=\widetilde{Q}_{2,0} e^{j \omega t}$, where $j$ is an imaginary unit and we consider that the real parts of complex quantities have physical meanings. If we put these expressions in Eqs. (11) and (12), we obtain a set of homogeneous linear equations in terms of $\widetilde{Q}_{1,0}$ and $\widetilde{Q}_{2,0}$. The characteristic equation is obtained by setting the determinant of the coefficient matrix of the linear equations to zero: 


$$
\begin{aligned}
& -\left(\frac{\rho L_{i}}{A_{i}} \frac{\rho L_{e}}{A_{e}} C\right)(j \omega)^{3}-\left[\frac{\rho \zeta_{T}}{A_{i}^{2}} \bar{Q} \frac{\rho L_{e} C}{A_{e}}-\frac{\rho L_{i}}{A_{i}} \frac{\rho C}{A_{e}^{2}}\left(D-\zeta_{2}\right) \bar{Q}\right. \\
& \left.+2 \rho C \alpha \frac{\cot \beta_{2}}{S}\left(\frac{\cot \beta_{2}}{S} \bar{Q}-U_{2}\right) \frac{\rho L_{e}}{A_{e}}\right](j \omega)^{2}+\left[-\frac{\rho L_{e}}{A_{e}}-\frac{\rho L_{i}}{A_{i}}\right. \\
& +\frac{\rho \zeta_{T}}{A_{i}^{2}} \frac{\rho C}{A_{e}^{2}}\left(D-\zeta_{2}\right) \bar{Q}^{2}-2 \rho C \alpha \frac{\cot \beta_{2}}{S}\left(\frac{\cot \beta_{2}}{S} \bar{Q}\right. \\
& \left.\left.-U_{2}\right) \frac{\rho\left(\zeta_{2}-D\right)}{A_{e}^{2}} \bar{Q}\right](j \omega)+\left[-\frac{\rho\left(\zeta_{2}-D\right)}{A_{e}^{2}} \bar{Q}-\frac{\rho \zeta_{T}}{A_{i}^{2}} \bar{Q}\right]=0
\end{aligned}
$$

Equation (13) is a third order equation in terms of $j \omega$ with real coefficients. From the characteristic equation (13), we can determine the complex frequency $\omega=\omega_{R}+j \omega_{I}$. The expression $e^{j \omega t}$ $=e^{j \omega_{R} t} \cdot e^{-\omega_{I} t}$ shows that the real part $\omega_{R}$ gives the frequency and the imaginary part $\omega_{I}$ gives the damping rate.

By taking the complex conjugate of Eq. (13), we can show that if $\omega_{1}=\omega_{1 R}+j \omega_{1 I}$ is a solution of Eq. (13), then $\omega_{2}=-\omega_{1 R}+j \omega_{1 I}$ is another solution. The solutions $\omega_{1}$ and $\omega_{2}$ are practically the same solutions with the same frequency $\omega_{1 R}$ and the common damping $\omega_{1 I}$. This also requires that the real part of the third solution be zero $\left(\omega_{3 R}=0\right)$. So, the solutions can be expressed as $\omega_{1}=\omega_{1 R}$ $+j \omega_{1 I}, \omega_{2}=-\omega_{1 R}+j \omega_{1 I}$, and $\omega_{3}=j \omega_{3 I}$.

Since Eq. (13) is a third order equation in terms of $j \omega$ with real coefficients, Hurwitz's criterion can be applied to obtain the stability condition:

$$
\begin{gathered}
{\left[\frac{\zeta_{T}}{A_{i}^{2}} \bar{Q} \frac{L_{e}}{A_{e}}-\frac{L_{i}}{A_{i}} \frac{D-\zeta_{2}}{A_{e}^{2}} \bar{Q}+2 \alpha \frac{\cot \beta_{2}}{S}\left(\frac{\cot \beta_{2}}{S} \bar{Q}-U_{2}\right) \frac{L_{e}}{A_{e}}\right] \times\left[\frac{L_{e}}{A_{e}}\right.} \\
+\frac{L_{i}}{A_{i}}-\frac{\zeta_{T}}{A_{i}^{2}} \frac{\rho C}{A_{e}^{2}}\left(D-\zeta_{2}\right) \bar{Q}^{2}-2 \rho C \alpha \frac{\cot \beta_{2}}{S}\left(\frac{\cot \beta_{2}}{S} \bar{Q}\right. \\
\left.\left.\quad-U_{2}\right) \frac{\left(D-\zeta_{2}\right)}{A_{e}^{2}} \bar{Q}\right]>\frac{L_{i}}{A_{i}} \frac{L_{e}}{A_{e}}\left[\frac{\zeta_{2}-D}{A_{e}^{2}}+\frac{\zeta_{T}}{A_{i}^{2}}\right] \bar{Q}
\end{gathered}
$$

Equation (14) can be used to determine the stable/unstable flow rate regions. However, the equation is rather complicated and we discuss the stability mainly based on the direct solution of the characteristic equation (13).

\section{Causes of Instability}

3.1 Diffuser Effect of the Draft Tube. Equation (12) can be written as

$$
\begin{aligned}
& \rho C \frac{L_{e}}{A_{e}} \frac{d^{2} \widetilde{Q}_{2}}{d t^{2}}+\rho C \frac{\zeta_{2}-D}{A_{e}^{2}} \bar{Q} \frac{d \widetilde{Q}_{2}}{d t}+\widetilde{Q}_{2} \\
& \quad=\widetilde{Q}_{1}+2 \rho C \alpha \frac{\cot \beta_{2}}{S}\left(\frac{\cot \beta_{2}}{S} \bar{Q}-U_{2}\right) \frac{d \widetilde{Q}_{1}}{d t}
\end{aligned}
$$

If we consider the case of $\widetilde{Q}_{1}=0$, negative damping occurs when

$$
D>\zeta_{2}
$$

This is caused by the diffuser effect of the draft tube. The frequency is given by

$$
\omega_{e}=\sqrt{\frac{A_{e}}{\rho L_{e} C}}
$$

This mechanism can be explained as follows. We consider the case when $Q_{2}$ is increased. From Eq. (3), $p_{a}$ will decrease if $D$ $>\zeta_{2}$ due to the diffuser effect, and the cavity volume $V_{c}$ is increased. Then, Eq. (1) shows that $Q_{2}$ is increased further if $Q_{1}$ is kept constant. This positive feedback is the cause of the instability due to the diffuser effect.

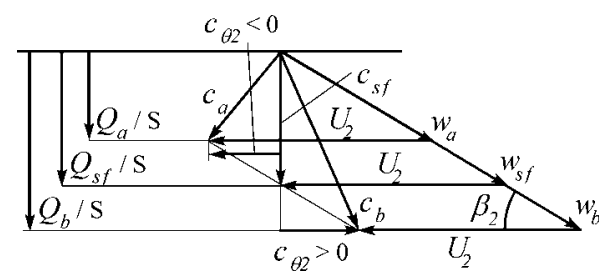

Fig. 2 Velocity triangle at the runner exit for three flow rates

3.2 Swirl Effect. We differentiate Eq. (11) with $t$ and represent $d^{2} \widetilde{Q}_{2} / d t^{2}$ with other terms. By putting it into Eq. (12), we obtain the following equation:

$$
\begin{aligned}
& \rho C \frac{L_{i}}{A_{i}} \frac{d^{2} \tilde{Q}_{1}}{d t^{2}}+\left[\rho C \frac{\zeta_{T}}{A_{i}^{2}} \bar{Q}+2 \rho C \alpha \frac{\cot \beta_{2}}{S}\left(\frac{\cot \beta_{2}}{S} \bar{Q}-U_{2}\right)\right] \frac{d \widetilde{Q}_{1}}{d t}+\tilde{Q}_{1} \\
& \quad=\widetilde{Q}_{2}
\end{aligned}
$$

Here, we consider the case with $\widetilde{Q}_{2}=0$. The first term of the multiplier on $d \widetilde{Q}_{1} / d t$ shows the damping caused by the resistance of the runner. The second term representing the effect of swirl becomes negative or positive depending on the value of tangential velocity $c_{\theta 2}=c_{m 2} \cot \beta_{2}-U_{2}=(\bar{Q} / S) \cot \beta_{2}-U_{2}$. The tangential velocity becomes zero at the flow rate $Q_{\mathrm{sf}}=S U_{2} \tan \beta_{2}$ and this is called the swirl-free flow rate. At a flow rate smaller than the swirl-free flow rate $Q_{\text {sf }}$, the swirl causes instability by reducing the damping coefficient. At a larger flow rate than the swirl-free flow rate, the swirl enhances damping. These effects can be explained as follows.

The velocity triangle at the runner exit is shown in Fig. 2 for three flow rates $Q_{a}, Q_{\mathrm{sf}}$, and $Q_{b}$. At smaller flow rate $Q_{a}$, the absolute value of the tangential velocity will decrease if the flow rate $Q_{a}$ is increased. Then, the pressure in the vortex core will increase and the cavity volume is decreased. The continuity equation (1) shows that the inlet flow rate $Q_{a}$ is increased further. This positive feedback is the cause of the instability. At larger flow rate $Q_{b}$ the tangential velocity increases if the flow rate is increased. Then, the core pressure is decreased and the cavity volume is increased. This results in the decrease in the upstream flow rate $Q_{1}$. This negative feedback is the cause of the stabilizing effect at a higher flow rate.

3.3 Energy Balance. We consider the displacement work of the cavitation region,

$$
E=\int p_{a}\left(Q_{2}-Q_{1}\right) d t=\int p_{a} Q_{2} d t-\int p_{a} Q_{1} d t=E_{2}-E_{1}
$$

under a steady oscillation condition. The pressure $p_{a}$ near the cavitation region can be evaluated from Eq. (2) or (3). Here, we separate each quantity $p_{a}$ into steady $\bar{p}_{a}$ and unsteady $\widetilde{p}_{a 0} e^{j \omega t}$ components and assume $\tilde{p}_{a 0} \ll \bar{p}_{a}$. We put $Q_{1}=\bar{Q}_{1}+\widetilde{Q}_{10} e^{j \omega t}$ in Eq. (2) and obtain

$$
\begin{gathered}
\bar{p}_{a}=p_{\text {inlet }}-\rho \frac{\zeta_{T}}{2 A_{i}^{2}} \bar{Q}^{2} \\
\tilde{p}_{a}=-\rho \frac{L_{i}}{A_{i}} j \omega \tilde{Q}_{1}-\rho \frac{\zeta_{T}}{A_{i}^{2}} \bar{Q}_{1} \tilde{Q}_{1}
\end{gathered}
$$

and Eq. (3) leads to

$$
\bar{p}_{a}=p_{\text {exit }}+\rho \frac{\zeta_{2}-D}{2 A_{e}^{2}} \bar{Q}_{2}^{2}
$$




$$
\tilde{p}_{a}=\rho \frac{L_{e}}{A_{e}} j \omega \tilde{Q}_{2}+\rho \frac{\zeta_{2}-D}{A_{e}^{2}} \bar{Q}_{2} \tilde{Q}_{2}
$$
$T$,

First, we consider the upstream energy transfer within a period

$$
\begin{aligned}
E_{1} & =\int_{0}^{T} p_{a} Q_{1} d t=\int_{0}^{T}\left(\bar{p}_{a}+\tilde{p}_{a}\right)\left(\bar{Q}_{1}+\widetilde{Q}_{1}\right) d t=\bar{p}_{a} \bar{Q}_{1} T+\int_{0}^{T} \tilde{p}_{a} \tilde{Q}_{1} d t \\
& =\bar{E}_{1}+\widetilde{E}_{1}
\end{aligned}
$$

In the same way,

$$
E_{2}=\int_{0}^{T} p_{a} Q_{2} d t=\bar{p}_{a} \bar{Q}_{2} T+\int_{0}^{T} \widetilde{p}_{a} \widetilde{Q}_{2} d t=\bar{E}_{2}+\widetilde{E}_{2}
$$

We consider the unsteady displacement work $\widetilde{E}_{1}$ and $\widetilde{E}_{2}$.

Using expression (21), we obtain the upstream work $\widetilde{E}_{1}$ as follows:

$$
\begin{aligned}
\widetilde{E}_{1} & =\int_{0}^{T} \widetilde{p}_{a} \widetilde{Q}_{1} d t=-\rho \frac{L_{i}}{A_{i}} \int_{0}^{T}\left(j \omega \widetilde{Q}_{1}\right) \widetilde{Q}_{1} d t-\rho \frac{\zeta_{T}}{A_{i}^{2}} \bar{Q}_{1} \int_{0}^{T} \widetilde{Q}_{1} \widetilde{Q}_{1} d t \\
& =-\rho \frac{\zeta_{T}}{A_{i}^{2}} \bar{Q}_{1}\left|\widetilde{Q}_{10}\right|^{2} \frac{\pi}{\omega}
\end{aligned}
$$

By using Eq. (23), we obtain

$$
\tilde{E}_{2}=\rho \frac{\zeta_{2}-D}{A_{e}^{2}} \bar{Q}_{2}\left|\widetilde{Q}_{20}\right|^{2} \frac{\pi}{\omega}
$$

So,

$$
\widetilde{E}=\widetilde{E}_{2}-\widetilde{E}_{1}=\rho \bar{Q} \frac{\pi}{\omega}\left(\frac{\zeta_{2}-D}{A_{e}^{2}}\left|\widetilde{Q}_{20}\right|^{2}+\frac{\zeta_{T}}{A_{i}^{2}}\left|\widetilde{Q}_{10}\right|^{2}\right)
$$

This means that the displacement work is dissipated by the upstream and downstream resistances.

The above discussions are based on the dynamics of the upstream and downstream flow channels. Equations (21) and (23) do not include the dynamics of cavitation. To clarify the contribution of cavitation, we use the continuity equation under cavitation, Eq. (12),

$$
\begin{aligned}
\tilde{Q}_{2}-\tilde{Q}_{1}= & -\rho C \frac{L_{e}}{A_{e}} \frac{d^{2} \tilde{Q}_{2}}{d t^{2}}+\rho C \frac{D-\zeta_{2}}{A_{e}^{2}} \bar{Q} \frac{d \tilde{Q}_{2}}{d t} \\
& +2 \rho C \alpha \frac{\cot \beta_{2}}{S}\left(\frac{\cot \beta_{2}}{S} \bar{Q}-U_{2}\right) \frac{d \tilde{Q}_{1}}{d t}
\end{aligned}
$$

By appropriately using Eqs. (21) and (23), we obtain

$$
\begin{aligned}
\widetilde{E}= & \int_{0}^{T} \widetilde{p}_{a}\left(\widetilde{Q}_{2}-\widetilde{Q}_{1}\right) d t=-\rho \frac{L_{i}}{A_{i}} 2 \rho C \alpha \frac{\cot \beta_{2}}{S}\left(\frac{\cot \beta_{2}}{S} \bar{Q}_{1}\right. \\
& \left.-U_{2}\right) \omega^{2} \frac{\pi}{\omega}\left|\widetilde{Q}_{10}\right|^{2}
\end{aligned}
$$

By equating Eqs. (28) and (30), we obtain

$$
\begin{aligned}
\rho \bar{Q} & \frac{\pi}{\omega}\left(\frac{\zeta_{2}-D}{A_{e}^{2}}\left|\tilde{Q}_{20}\right|^{2}+\frac{\zeta_{T}}{A_{i}^{2}}\left|\tilde{Q}_{10}\right|^{2}\right) \\
& =-\rho \frac{L_{i}}{A_{i}} 2 \rho C \alpha \frac{\cot \beta_{2}}{S}\left(\frac{\cot \beta_{2}}{S} \bar{Q}-U_{2}\right) \omega^{2} \frac{\pi}{\omega}\left|\widetilde{Q}_{10}\right|^{2}
\end{aligned}
$$

Equation (31) shows the following.

(1) The displacement work by the cavity due to swirl shown by the right hand side should be dissipated by the resistance in the upstream and downstream.
Table 1 Solutions of characteristic equations for the standard case

Third order equation, Eq. (13)

$$
\begin{array}{cl}
\omega_{1,2}= \pm 13.14-5.17 j & \widetilde{Q}_{1,0} / \widetilde{Q}_{2,0}=-0.0217 \mp 0.018 j \\
\omega_{3}=0+1.88 j & \widetilde{Q}_{1,0} / \widetilde{Q}_{2,0}=-0.303 \mp 0 j
\end{array}
$$

Second order equation, Eq. (15)

$$
\omega_{1,2}= \pm 12.33-2.34 j \quad \widetilde{Q}_{1,0} / \widetilde{Q}_{2,0}=-0.0228 \mp 0.015 j
$$

(2) With $D=\zeta_{2}$, steady oscillation is possible only for $\bar{Q}_{1}$ $<S U_{2} \tan \beta_{2}$.

(3) With $\alpha=0$, steady oscillation is possible only for $D=\zeta_{2}$ $+A_{e}^{2} / A_{i}^{2}\left|\widetilde{Q}_{10} / \widetilde{Q}_{20}\right|^{2} \zeta_{T}$.

(4) The cavity provides energy only through the swirl flow effects. With the diffuser effects, the energy is provided by the diffuser and the cavity does not contribute to the energy supply. The cavity is needed only for constituting a vibration system.

\section{Numerical Results}

The values of the parameters used for sample calculations are given in the nomenclature. These values are determined by considering a test facility at EPFL and used for sample calculations as standard values except for the parameters specified for each case. The value of the loss coefficient $\zeta_{T}$ representing the effect of the runner was determined by assuming that the applied head $H$ equals the loss head $\left(\zeta_{T} / 2 g\right)\left(\bar{Q} / A_{i}\right)^{2}$ across the runner. The value of the cavitation compliance $C$ was determined so that the frequency given by Eq. (17) becomes 0.16 times the rotational frequency of the impeller, $f_{n}$.

The swirl-free flow rate $Q_{\text {sf }}$, which gives no swirl at the runner exit, is calculated to be

$$
Q_{\text {sf }}=S U_{2} \tan \beta_{2}=0.618 \mathrm{~m}^{3} / \mathrm{s}
$$

5.1 Mode of Oscillation. The ratio of the amplitudes of the flow rate fluctuation in the upstream and downstream can be determined from Eq. (11) to be

$$
\frac{\widetilde{Q}_{1,0}}{\widetilde{Q}_{2,0}}=-\left[\frac{\rho\left(\zeta_{2}-D\right)}{A_{e}^{2}} \bar{Q}+\frac{\rho L_{e}}{A_{e}} j \omega\right] /\left[\frac{\rho \zeta_{T}}{A_{i}^{2}} \bar{Q}+\frac{\rho L_{i}}{A_{i}} j \omega\right]
$$

Since $\left(\zeta_{2}-D\right) / A_{e}^{2} \ll \zeta_{T} / A_{i}^{2}$ and $L_{e} \ll L_{i}$, inlet flow rate fluctuation $\widetilde{Q}_{1}$ is much smaller than the outlet flow rate fluctuation $\widetilde{Q}_{2}$. In this case, Eq. (15) with $\widetilde{Q}_{1}=0$ is expected to give a good approximation.

Table 1 shows the result for the standard case. Third order equation shows the results from Eq. (13) and second order equation shows the results from Eq. (15) with $\widetilde{Q}_{1}=0$. The third order equation has solutions $\omega_{1}$ and $\omega_{2}$ with the same imaginary part, and positive and negative real parts with the same absolute value. The real part of $\omega_{3}$ is zero and the imaginary part is positive, suggesting an exponentially decaying mode. This shows that the third order characteristic equation (13) gives substantially only one oscillatory mode. As expected, $\left|\widetilde{Q}_{1,0} / \widetilde{Q}_{2,0}\right|$ is much smaller than 1 for $\omega_{1}$ and $\omega_{2}$. The second order equation (15) also gives the solutions $\omega_{1}$ and $\omega_{2}$ with positive and negative real parts with the same absolute value. We discuss about the absolute value of the real part of $\omega_{1}$ and $\omega_{2}$ as the frequency and the common imaginary part of $\omega_{1}$ and $\omega_{2}$ as the damping rate. The difference of the values of $\omega_{1}$ and $\omega_{2}$ from the second and third equations is not as small as expected from the small value of $\left|\widetilde{Q}_{1,0} / \widetilde{Q}_{2,0}\right|$. This 

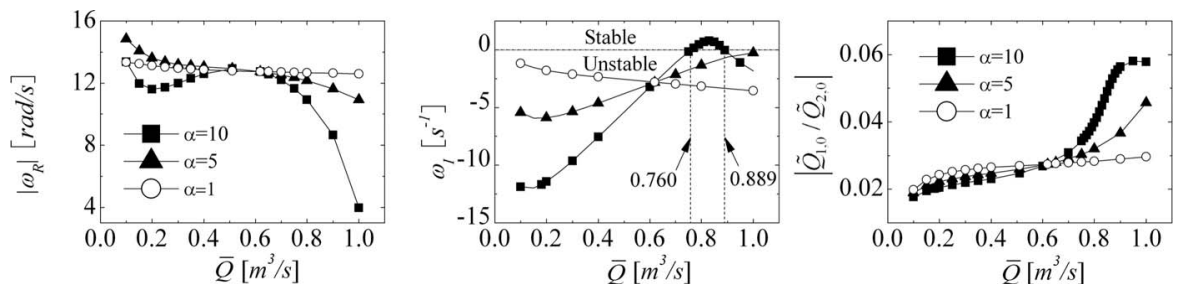

Fig. 3 Effects of mean flow rate $\bar{Q}$ under standard conditions

shows that the swirl has a significant effect even if the upstream flow rate fluctuation is small, perhaps caused by larger values of $\cot \beta_{2}=3.17$ and $\alpha=10$.

\subsection{Effects of Mean Flow Rate and Contributions of Dif-} fuser and Swirl Effects. A large effect of mean flow rate $\bar{Q}$ on the instability is expected from the discussion in the last section. So, the effect of flow rate is examined from the numerical results from Eq. (13). Here, the head is assumed to be constant and the mean flow rate $\bar{Q}$ is changed by changing the value of $\zeta_{T}$. Figure 3 shows the results with three values of the pressure coefficient of swirl $\alpha$. With the standard value $\alpha=10$, the instability occurs for $\bar{Q}<0.760 \mathrm{~m}^{3} / \mathrm{s}$ or $\bar{Q}>0.889 \mathrm{~m}^{3} / \mathrm{s}$, which includes the standard flow rate $Q_{s}=0.51 \mathrm{~m}^{3} / \mathrm{s}$ and the swirl-free flow rate $Q_{\mathrm{sf}}$ $=0.618 \mathrm{~m}^{3} / \mathrm{s}$. For $\bar{Q}<0.76 \mathrm{~m}^{3} / \mathrm{s}$, the value of $\left|\widetilde{Q}_{1,0} / \widetilde{Q}_{2,0}\right|$ is smaller but it increases significantly for $\bar{Q}>0.889 \mathrm{~m}^{3} / \mathrm{s}$. With smaller values of $\alpha=5$ and $\alpha=1, \omega_{I}$ is negative for all flow rates $\widetilde{Q}$ shown. To understand this result, diffuser effects and the swirl effects are examined independently.

First, calculations were made with $\alpha=0$ to examine the diffuser effects and the results are shown in Fig. 4 with various values of the diffusion factor $D . D_{\text {stand }}=27.7$ is the standard value of the diffusion factor. We should note that small but positive damping $\omega_{I}>0$ is obtained for the case of $D-\zeta_{2}=0$. This shows that the diffuser effect represented by $D$ is the cause of the instability. For $D=D_{\text {stand }}$ and $D=0.5 D_{\text {stand }}$, the instability occurs for all flow rates and the amplifying rate $-\omega_{I}$ increases with the flow rate and the value of the diffusion factor $D$. This is expected from the damping term in Eq. (15).

Second, the effect of swirl is examined by setting $D-\zeta_{2}=0$ and the results with several values of swirl pressure coefficient $\alpha$ are shown in Fig. 5. Equation (18) with $\widetilde{Q}_{2}=0$ suggests that the up- stream resonant frequency $\omega_{i}=\sqrt{A_{i} / \rho L_{i} C}=2.13 \mathrm{rad} / \mathrm{s}$. However, the frequency is closer to the downstream resonant frequency $\omega_{e}$ $=\sqrt{A_{e} / \rho L_{e} C}=12.56 \mathrm{rad} / \mathrm{s}$ of Eq. (17).

The critical flow rate at which the damping coefficient on $d \widetilde{Q}_{1} / d t$ in Eq. (18) becomes zero is obtained to be $\bar{Q}$ $=0.569 \mathrm{~m}^{3} / \mathrm{s}$. However, the critical flow rate with $\omega_{I}=0$ shown in Fig. 5 is closer to the swirl-free flow rate $Q_{\text {sf }}=0.618 \mathrm{~m}^{3} / \mathrm{s}$ irrespective of the value of $\alpha$. The damping rate $\omega_{I}$ is nearly proportional to the value of $\alpha$. For the case of $D-\zeta_{2}=0$, Hurwitz's criterion (14) reduces to

$$
\bar{Q}>\frac{2 \alpha U_{2} \cot \beta_{2} / S}{\frac{\zeta_{T}}{A_{i}^{2}}+2 \alpha\left(\frac{\cot \beta_{2}}{S}\right)^{2}-\left(\frac{\zeta_{T}}{A_{i}^{2}} \frac{L_{i}}{A_{i}}\right) /\left(\frac{L_{e}}{A_{e}}+\frac{L_{i}}{A_{i}}\right)}
$$

For the present case with $L_{i} / A_{i} \gg L_{e} / A_{e}$, the third term in the denominator on the right hand side almost cancels the first term and Eq. (20) can be approximated by

$$
\bar{Q}>\frac{2 \alpha U_{2} \cot \beta_{2} / S}{2 \alpha\left(\cot \beta_{2} / S\right)^{2}}=S U_{2} \tan \beta_{2}=Q_{\mathrm{sf}}
$$

These results show that in real cases with $L_{i} / A_{i} \gg L_{e} / A_{e}$, the swirl effect causes the instability at smaller flow rates than the swirl-free flow rate irrespective of the larger loss coefficient $\zeta_{T}$ of the runner. This result is in good agreement with Dorfler's study [9], which indicates that the self-excited surge can also occur at a low flow rate. The amplitude of the upstream flow rate fluctuation is much smaller and the frequency is closer to the downstream resonant frequency $\omega_{e}$.

If we consider the case with $D-\zeta_{2}=0$ and $\zeta_{T}=0$, the characteristic equation (13) is reduced to
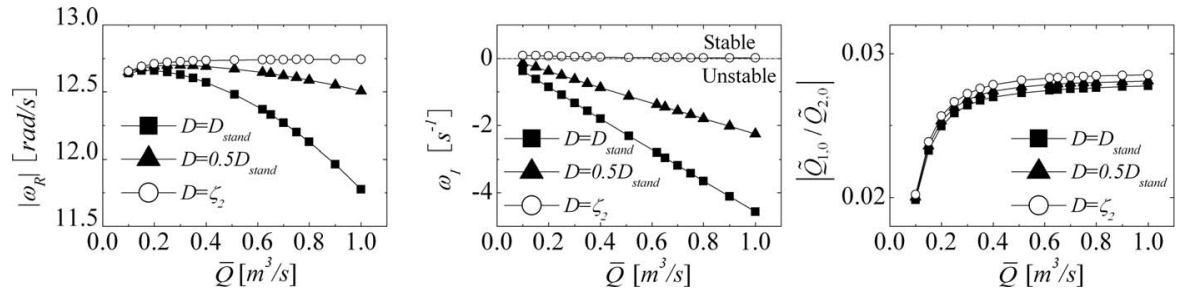

Fig. 4 Effects of diffusion factor $D$ under $\alpha=0$
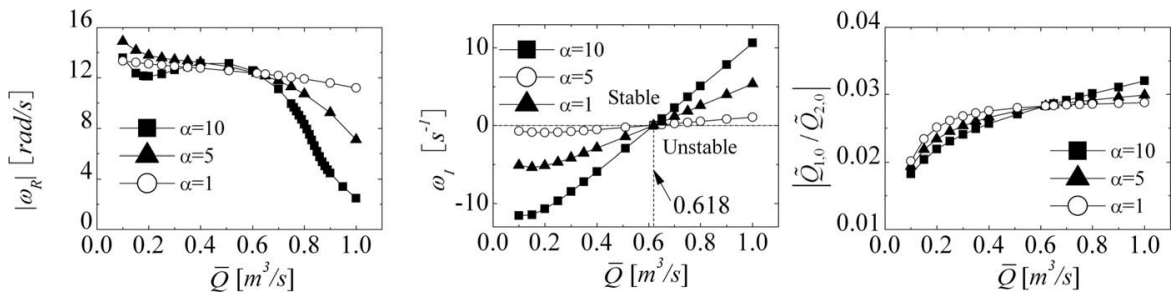

Fig. 5 Effects of pressure coefficient of swirl $\alpha$ under $D-\zeta_{2}=0$ 


$$
\begin{gathered}
-\left(\frac{\rho L_{i}}{A_{i}} \frac{\rho L_{e}}{A_{e}} C\right) \omega^{2}+j\left[\frac{2 \rho C \alpha \cot \beta_{2}}{S}\left(\frac{\cot \beta_{2}}{S} \bar{Q}-U_{2}\right) \frac{\rho L_{e}}{A_{e}}\right] \omega \\
+\left[\frac{\rho L_{e}}{A_{e}}+\frac{\rho L_{i}}{A_{i}}\right]=0
\end{gathered}
$$

This equation shows that the damping is caused by the swirl effects and the resonant frequency is given by

$$
\omega_{r}=\sqrt{\frac{L_{e} / A_{e}+L_{i} / A_{i}}{\rho C\left(L_{i} / A_{i}\right)\left(L_{e} / A_{e}\right)}}
$$

We obtain the downstream resonance with $\omega_{r}=\omega_{e}=\sqrt{A_{e} / \rho C L_{e}}$ for $L_{i} / A_{i} \gg L_{e} / A_{e}$ and the upstream resonance with $\omega_{r}=\omega_{i}$ $=\sqrt{A_{i} / \rho C L_{i}}$ for $L_{i} / A_{i} \ll L_{e} / A_{e}$. This shows that the swirl effect can cause both upstream and downstream flow oscillations although the upstream flow rate fluctuation is essential in the positive feedback loop of the instability through the swirl.

In order to discuss about the combined effects of the diffusion and the swirl, we compare the results shown in Figs. 3-5. The similarity of the plots of $\omega_{R}$ in Figs. 3 and 5 shows that the swirl has the most significant effects on the frequency $\omega_{R}$ under standard conditions. The comparison of the plots of $\omega_{I}$ shows that $\omega_{I}$ for the standard case in Fig. 3 approximately equals the sum of those in Fig. 4 with $D=D_{\text {stand }}$ and in Fig. 5 except for the case of $\alpha=10$ with a larger value of $\bar{Q}$. The examination of $\left|\widetilde{Q}_{1,0} / \widetilde{Q}_{2,0}\right|$ in each figure shows that the value is generally small but relatively larger values are obtained at larger values of $\bar{Q}$ with $\alpha=10$ for the general case shown in Fig. 3. This and the behavior of $\omega_{I}$ in Fig. 3 for the case of $\alpha=10$ with larger value of $\bar{Q}$ are considered to be a result of the combined effects of diffusion and swirl.

\section{Conclusion}

It was found that the diffuser effect of the draft tube destabilizes the hydraulic system over the entire flow range. The swirl flow from the runner stabilizes/destabilizes the system above/below the swirl-free flow rates. In both cases, the frequency of oscillation is determined from the compliance of the cavitation and the inertial length of the draft tube. For general cases with larger penstock length and runner resistance, the amplitude of the flow rate fluctuation is much larger in the downstream of the runner as compared to that in the upstream. The analysis can be applied not only to the full load surge but also to the part load surge, which has been considered to be a forced oscillation due to the vortex rope whirl.

Further research is needed to correlate these findings with experimental observations in real hydropower systems. Various simplifying assumptions have been made in this study. For the application to a real system, we need to take into account the flow compressibility effects in the penstock. For lower frequency oscillations, we may need to take into account the changes in the runner speed and guide vane opening. Swirl in the draft tube may mitigate the flow separation and thus reduce the value of draft tube resistance $\zeta_{2}$ [8]. At a higher mean flow rate than design, this effect might destabilize the system, and the stability would be enhanced at lower flow than design. These effects should be clarified in future study.

\section{Acknowledgments}

The authors would like to express their sincere gratitude to Professor Michihiro Nishi at Kyusyu Institute of Technology, Mr.
Hiroshi Kato of DMW Corporation, and Professor Takashi Kubota of South China University of Technology for their fruitful discussions.

\section{Nomenclature}

\section{Standard Value}

$$
\begin{aligned}
A_{c}=0.125 \mathrm{~m}^{2} & =\text { draft tube inlet area } \\
A_{i}=0.22 \mathrm{~m}^{2} & =\text { inlet pipe area } \\
A_{e}=0.67 \mathrm{~m}^{2} & =\text { draft tube exit area } \\
C=97.2 \times 10^{-7} \mathrm{~m}^{4} \mathrm{~s}^{2} / \mathrm{kg} & =\text { cavitation compliance } \\
c_{2} & =\text { runner exit absolute velocity } \\
c_{m 2} & =\text { runner exit meridional velocity } \\
c_{\theta 2} & =\text { runner exit tangential velocity } \\
D=\left(A_{e} / A_{c}\right)^{2}-\mathrm{I}=27.7 & =\text { diffusion factor of draft tube } \\
d=0.4 \mathrm{~m} & =\text { runner exit diameter } \\
f_{n}=12.5 \mathrm{~Hz} & =\text { rotational speed } \\
H=14.8 \mathrm{~m} & =\text { head } \\
L_{e}=4.36 \mathrm{~m} & =\text { effective length of draft tube } \\
L_{i}=50 \mathrm{~m} & =\text { inlet pipe length } \\
p & =\text { pressure } \\
Q=0.51 \mathrm{~m}^{3} / \mathrm{s} & =\text { flow rate } \\
Q_{\mathrm{sf}}=0.618 \mathrm{~m}^{3} / \mathrm{s} & =\text { swirl-free flow rate } \\
\bar{Q} & =\text { steady part of flow rate } \\
\widetilde{Q} & =\text { unsteady part of flow rate } \\
S_{2}=0.125 \mathrm{~m}^{2} & =\text { runner exit area } \\
U_{2}=15.7 \mathrm{~m} / \mathrm{s} & =\text { runner exit circumferential } \\
V_{c} & =\text { velocity } \\
\alpha=10 & =\text { prelume of cavity } \\
\beta_{2}=17.5 \mathrm{deg} & =\text { runner exit blade angle } \\
\rho=1000 \mathrm{~kg} / \mathrm{m}^{3} & =\text { fluid density } \\
\omega=\omega_{R}+j \omega_{I} & =\text { complex frequency } \\
\zeta_{2}=0.207 & =\text { loss coefficient of draft tube } \\
\zeta_{T}=54.2 & =\text { runner resistance } \\
&
\end{aligned}
$$

\section{References}

[1] Jacob, T., and Prenat, J.-E., 1996, "Francis Turbine Surge: Discussion and Data Base," Proceedings of the 18th IAHR Symposium, Valencia, Spain.

[2] Nishi, M., 1984, "Surging Characteristics of Conical and Elbow Type Draft Tubes," Proceedings of the 12th IAHR Symposium on Hydraulic Machinery and System, Stirling, U.K., pp. 272-283.

[3] Nishi, M., Matsunaga, S., Kubota, T., and Senoo, Y., 1982, "Flow Regimes in an Elbow-Type Draft Tube," Proceedings of the 11th IAHR Symposium on Hydraulic Machinery and System, Amsterdam, Netherlands, Paper No. 38, pp. $1-13$.

[4] Nishi, M., Wang, X., Okamoto, M., and Matsunaga, S., 1994, "Further Investigation on the Pressure Fluctuations Caused by Cavitated Vortex Rope in an Elbow Draft Tube," Cavitation and Gas Fluid Flow Machinery and Devices, ASME, pp. 63-70.

[5] Prenat, J.-E., and Jacob, T., 1986, "Investigating the Behavior at High Load of a Francis Turbine Model," Proceedings of the 13th IAHR Symposium, Montreal, Canada.

[6] Koutnik, J., and Pulpitel, L., 1996, "Modeling of the Francis Turbine FullLoad Surge," Modeling, Testing and Monitoring for Hydro Power Plants, Lausanne, Switzerland.

[7] Koutnik, J., Nicolet, C. A., Schoul, G., and Avellan, F., 2006, "Overload Surge Event in a Pumped Storage Power Plant," Proceedings of the 23rd IAHR Symposium, Yokohama, Japan, Paper No. 135.

[8] Susan-Resiga, R., Ciocan, G. D., Anton, I., and Avellan, F., 2006, "Analysis of the Swirling Flow Downstream a Francis Turbine Runner," ASME J. Fluids Eng., 128, pp. 177-189.

[9] Dorfler, P. K., 1985, "Francis Turbine Surge Prediction and Prevention," Proceedings of the Waterpower '85, pp. 952-961. 